\title{
Modifikation eines Modells von Verbindungsmitteln zur Prognose des Schalldämmmaßes von Leichtbaukonstruktionen mittels Finite Elemente-Methode im erweiterten Frequenzbereich
}

\author{
M. Neusser (D, T. Bednar $(\mathbb{0}$
}

\begin{abstract}
Die vorliegende Arbeit hat die primäre Zielsetzung, ein leistungsfähiges Berechnungsmodell für die Prognose des Schwingungsverhaltens und damit des Schalldämmmaßes von „leichten“ Baukonstruktionen zu entwickeln. Eine Anwendung der derzeit normativ abgedeckten Verfahren für diese Baukonstruktionen ist ausdrücklich in den betreffenden Regelwerken ausgeschlossen. Die derzeit auf wissenschaftlicher Basis stehenden Berechnungsverfahren bieten im betrachteten Frequenzbereich zwischen $50 \mathrm{~Hz}$ und $5000 \mathrm{~Hz}$ keine ausreichende Zuverlässigkeit der Prognosequalität in den schalltechnischen Kenngrößen zur Beschreibung des bauakustischen Verhaltens solcher Konstruktionen. Neben den zu erwarteten diffusen Berechnungsergebnissen im unteren Frequenzspektrum ist eine Abbildung der Verbindungsausbildung zwischen Plattenwerkstoff und Tragkonstruktion, die in dem betreffenden Frequenzbereich einen wesentlichen Einfluss aufweisen, nicht möglich. Ebenso ist es daher auch nicht möglich, mittels bestehender Verfahren die Auswirkungen von schwankender Verarbeitungsqualität, wie z. B. den Schraubenanzugsmoment, in die Prognose der Kenngrößen einfließen zu lassen. Das entwickelte Simulationsmodell in der Finite Elemente-Umgebung "COMSOL 5.6" bietet die Möglichkeit der Berücksichtigung der Parameter Schraubendimensionen, Schraubenabstand, Anzugsmoment und Position der Schrauben auf der Tragkonstruktion. Durch die numerischen Ergebnisse des Prognosemodells können somit die Wechselwirkungen zwischen den unterschiedlichen Wandkomponenten und deren Verbindungselementen untersucht und optimiert werden.
\end{abstract}

Schlüsselwörter: Finite Elemente-Methode; Schalldämmung; Leichtbau; Verbindungsmittel

Modification of a model of connection parts in the finite element method for the prediction of the sound transmission loss of lightweight constructions in the extended frequency range.

The primary aim of this thesis is to develop a high-performance calculation model for predicting vibration characteristics and consequent measures of the sound transmission loss of lightweight building structures. An application of the currently normative covered processes for these structures is explicitly excluded in the relevant body of standards. The current calculation models, based on scientific models, do not offer satisfactory reliability in the quality of predicting the acoustical performance characteristics - especially in the low frequency range - to describe the acoustical behavior of such structures. Besides the expected vague calculation results in the spectra of low frequencies, it is not possible to build an image of formations of the connections between the outer panels and the supporting structure, which can present an important factor in these frequency ranges. Also, with the current state-of-the-art simulation models it is not practicable to incorporate the effects of fluctuating quality of workmanship, such as panel fastening, into the body of rules with the current processes. These facts lead to over-dimensioning the building components to compensate for the until now mostly unknown phenomena and their unknown influence of the acoustical performance characteristics. The developed simulation model in the finite element environment "COMSOL 5.6" offers the possibility of considering the parameters in the connection joints such as screw dimensions, screw spacing, tightening torque and position of the screws on the supporting structure. The numerical results of the validated forecast model allow the interactions between the different wall components and their fasteners to be investigated and optimized.

Keywords: finite element method; sound transmission loss; leigthweight building constructions; connections

Eingegangen am 15. Jänner 2021, angenommen am 11. März 2021, online publiziert am 25. März 2021

() The Author(s) 2021

\section{Einleitung}

Die Urbanisierung und die wachsende Verdichtung von Wohnraum tragen dazu bei, dass Lärmbelästigung ein immer wesentlicheres Thema in der Gesellschaft einnimmt. [1] In aktuellen Studien der WHO wie zum Beispiel in „Burden of disease from environmental
Neusser, Maximilian, Institut für Werkstofftechnologie, Bauphysik und Bauökologie, Forschungsbereich Bauphysik, Technische Universität Wien, Karlsplatz 13, 1040 Wien, Österreich (E-Mail: maxmilian.neusser@tuwien.ac.at); Bednar, Thomas, Institut für Werkstofftechnologie, Bauphysik und Bauökologie, Forschungsbereich Bauphysik, Technische Universität Wien, Wien, Österreich 
noise. Quantification of healthy life years lost in Europe" von 2011 wird auf die direkte Kausalität der Lärmaussetzung von Menschen auf die Schlafqualität verwiesen und die daraus möglichen gesundheitlichen Folgen dargestellt. Die Vielfallt der Lärmquellen bedürfen immer umfassender Schutzmaßnahmen, um dem Schutzziel der Gesundheit und des Komforts der Nutzer von Gebäuden zu entsprechen. Um diese Schutzziele zu erreichen, hat der Gesetzgeber Anforderungen [2] an Gebäudebauteile definiert. Derzeit in Österreich gültige Deskriptoren zur Beschreibung der akustischen Performance vernachlässigen den tiefen Frequenzbereich und somit eine potentielle Schwachstelle von leichten Baukonstruktionen. Eine international geführte Diskussion im Rahmen der COST Action TU0901 zur Erweiterung der Deskriptoren im tiefen und hohen Frequenzbereich, stellt allerdings aus vielfältigen Gründen eine große Schwierigkeit für die Planer und die Industrie dar.

Vor allem für leichte Bauelemente, sei es aus mineralisch gebundenen Werkstoffen oder aus Holzwerkstoffen, die einen immer prominenteren Anteil der verbauten Baukonstruktionen darstellen [3], ist diese Entwicklung eine Herausforderung. Die wachsende Anwendung von derartigen Konstruktionen erweitert das Problem zunehmend, sodass aktuell normativ regulierte Rechenmodelle (z.B. EN ISO 12354-1) nicht in der Lage sind die bauakustische Performance und somit das besondere physikalische Verhalten solcher Konstruktionen zu prognostizieren. Die Prognose der bauakustischen Deskriptoren leichter Baukonstruktionen stellt somit eine Unsicherheit innerhalb von Forschung und Entwicklung dar. Aus derzeitiger Sicht eines Herstellers ist eine Messung zur Bestimmung der bauakustischen Kenngrößen jeder einzelner Bauteilvariante, die einzige Möglichkeit Planungs- und Entwicklungsfortschritte zu quantifizieren, um den Planern eine Beurteilung der Einhaltung der gesetzlichen Anforderungen durch die gewählte Konstruktion zu ermöglichen.

Leichte Baukonstruktionen bestehen in der Regel aus einer Tragkonstruktion und einem wandbildenden Bauelement, die beide mittels unterschiedlichen Konstruktionsmitteln tragfähig miteinander verbunden sind. In $[4,5]$ wurde gezeigt, dass die Einflüsse von sekundären Wanddetails von Leichtbautrennwänden einen wesentlichen Einfluss auf das Schalldämmmaß haben. Untersucht wurden die Einflüsse von Schraubenabstand, und Schraubenanordnung Diese Parameter werden wesentlich durch die Verarbeitungsqualität auf der Baustelle beeinflusst. Die Ausbildung dieser Verbindung stellt einen bis dato noch in den Planungsmethoden weitestgehend unbekannten Einflussfaktor auf die akustische Performance leichter Baukonstruktionen dar

Die Prognose des Luftschalldämmmaßes von Baukonstruktion wurde intensiv durch unterschiedliche Forschungsgruppen erforscht und es wurde eine Vielzahl von unterschiedlichen Modellen erstellt, die grundlegend auf verschiedenen Vorgehensweisen basieren. In [6] gibt einen Überblick über die bereits entwickelten Methoden Diese Arbeit beschäftigt sich mit Simulations Modellen die die FEM als Basis besitzen. Die Anwendung der FEM in der Bauakustik ist im Vergleich zu anderen Bereichen, z.B. der Strukturdynamik nich weit verbreitet, wobei ein besonderes Hindernis die Abbildung der Verbindungen zwischen den einzelnen Elementen einer Leichtbaukonstruktion darstellt.

Die Anwendung der Finite-Elemente-Methode für die Lösung des gekoppelten strukturellen akustischen Problems wird in der Literatur vielfältig diskutiert [7-15]. Eine konkrete Anwendung in der Bauakustik bietet das numerische FEM-Modell in [16]. Das Modell wurde verwendet, um das Schalldämmmaß von Einschaligen- und Doppelschaligen Wänden zu berechnen. Als wesentlichen Parameter der Studie befinden sich die Rolle der Steifigkeit und die Ausbildung der Tragkonstruktion bei niedrigeren Frequenzen im Fokus. Das Modell

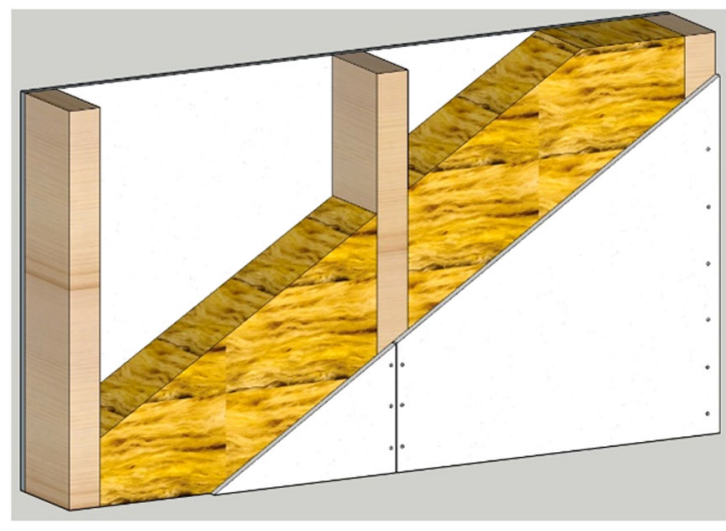

Abb. 1. Schematische Darstellung des untersuchten Bauteilaufbaus einer Gipskartonständerkonstruktion mit Holzstehern und Mineralwolleeinlage

konnte erfolgreich validiert werden und zeigt einen deutlichen Einfluss der Steher und dessen Geometrie auf das Schalldämmaß von doppelschaligen Ständerkonstruktionen auf. Der Einfluss der Verbindungsmittel wird nicht dargestellt und somit nicht in das Modell implementiert. Ein ähnliches Modell wurde in [17] entwickelt, das ebenso das Schalldämmmaß von doppelschaligen Konstruktionen erfolgreich prognostizieren kann, und fokussiert sich dabei auf die Modellierung in den Strukturen einer ungleichmäßig, über den Frequenzbereich verteilten mechanische Dämpfung der Schwingungsenergie. Das Modell berücksichtigt unter anderem die Wechselwirkung der mechanischen Verbindung zwischen Tragkonstruktion und Wandbildenden Plattenwerkstoffen. Die numerischen Prognosen stimmen gut mit den experimentellen Daten überein, wobei die Einzahlwerte von Simulation und Messung $\left(R_{w}, C\right.$ und $\left.C_{t r}\right)$ eine maximale Abweichung von $1 \mathrm{~dB}$ liefern. [17] Die besonders hohe erzielte Genauigkeit lässt sich auf die Anwendung eins iterativen Verfahrens zur Bestimmung der frequenzabhängigen Parameter des Dämpfungsmodells zurückführen, womit die Ergebnisse an die Messung gefittet werden.

Die FEM wurde somit bereits mehrfach erfolgreich zur Prognose des Schalldämmmaßes von Leichtbaukonstruktionen eingesetzt [16-18]. Alle diese Modelle haben allerdings gemein, dass sie einen oder mehrere der in [4,5] für das Schalldämmmaß als maßgeblichen bestimmten Parameter der Verbindung zwischen Steher und Platte nicht berücksichtigen. Diese Parameter wie Schraubenabstand, Schraubenposition, Schraubendimension und Schraubenanzugsmoment können das einzahlbewertete Schalldämmmaß von Leichtbaukonstruktionen um bis zu $10 \mathrm{~dB}$ verändern [5]. Ziel dieser Arbeit ist es die Modellierungsansätze aus [4, 16-18] um diese Parameter zu erweitern und aufbauend auf diesen Erkenntnissen ein Simulationsmodell zu entwickeln, dass diese Einflüsse wiedergeben und prognostizieren kann.

\section{Bauteil und Materialeigenschaften}

Im Fokus dieser Arbeit steht die Charakterisierung und Modellbildung von Bauteilverbindungselementen. Daher wurde ein Bauteilaufbau gewählt, der alle wesentlich Komponenten einer Leichtbauwand enthält, aber in seiner Komplexität einfach gehalten ist. Hierfür wurde eine Probekörperkonfiguration gewählt, die eine eindeutige Charakterisierung der Verbindung zwischen Plattenwerkstoff und Tragkonstruktion (z.B. der Schraube) erlaubt und dessen Einzelkomponenten eine einfache Modellelierung erlauben. Abbildung 1 zeigt eine schematische Darstellung der Probekörperkonfiguration 
Tab. 1. Materialparameter - Holz

\begin{tabular}{ll}
\hline E-Modul in N/mm & 8950 \\
Querkontraktionszahl & 0.3 \\
Rohdichte in $\mathrm{kg} / \mathrm{m}^{3}$ & 400 \\
Isotropischer Verlustfakor & $0.011($ bei $500 \mathrm{~Hz}$ ) \\
\hline
\end{tabular}

Tab. 2. Materialparameter - Gipsfaserplatte

\begin{tabular}{ll}
\hline E-Modul in N/mm & 4150 \\
Querkontraktionszahl & 0.18 \\
Rohdichte in $\mathrm{kg} / \mathrm{m}^{3}$ & 1200 \\
Isotropischer Verlustfakor & $0.014($ bei $500 \mathrm{~Hz}$ ) \\
\hline
\end{tabular}

\section{Tab. 3. Materialparameter - Schraube}

\begin{tabular}{ll}
\hline E-Modul in N/mm & \\
Querkontraktionszahl & 4150 \\
Rohdichte in $\mathrm{kg} / \mathrm{m}^{3}$ & 0.18 \\
Isotropischer Verlustfakor & 1200 \\
\hline
\end{tabular}

\section{Tab. 4. Materialparameter - Mineralfaser}

\begin{tabular}{ll}
\hline Dynamische Viskosität in Pa & $1.825 \mathrm{E}-10$ \\
Wärmeleitfähigkeit W/(m·K) & 0.04 \\
Rohdichte in $\mathrm{kg} / \mathrm{m}^{3}$ & 65 \\
Spezifische Wärmekapazität J/(kg.K) & 1000 \\
Porosität & 0.99 \\
Strömungswiderstand in Pa·s/m² & 8000 \\
Viskose charakteristische Länge in m & $1.92 \mathrm{E}-4$ \\
Thermische charakteristische Länge in m & $3.84 \mathrm{E}-4$ \\
\hline
\end{tabular}

bestehend aus einer Gipsfaserplatte in $15 \mathrm{~mm}$ Stärke und einem Holz Steher $(160 \mathrm{~mm} \times 60 \mathrm{~mm} \times 1230 \mathrm{~mm}$ ) welche miteinander verschraubt wurden. Der Prüfkörper hatte somit eine Außenabmessung von $1230 \mathrm{~mm} \times 1480 \mathrm{~mm} \times 190 \mathrm{~mm}$. Die Schrauben sind mittig auf den Stehern angeordnet und besitzen einen Abstand von $177 \mathrm{~mm}$. Der zwischen den beiden Plattenebenen entstehende Hohlraum wurde mit Mineralfaser vollständig ausgefüllt.

\subsection{Materialdaten}

Tabelle 1, 2, 3 und 4 zeigen die Materialdaten, die innerhalb der numerischen Simulation verwendet wurden. Die Ermittlung dieser Materialdaten erfolgte anhand von unterschiedlichen Versuchsaufbauten und ist in [4] dargestellt.

\section{Methodik}

Ziel der präsentierten Arbeit ist die Erstellung eines FEM basierenden Simulationsmodells in der Software Umgebung "COMSOL 5.6" der in Abschn. 2 beschriebenen Wandkonstruktion. Insbesondere liegt der Fokus dabei auf der Modellierung der Schraubverbindung und die Berücksichtigung der für die Prognose des Schalldämmmaßes solcher Konstruktionen notwendigen Parameter. Als Basis für die Erstellung eines auf einer Finiten Elemente Methode (FEM) basierenden Simulationsmodells dienen die Messungen und Erkenntnisse aus [4, 5]. Der nun folgende Abschnitt beschreibt die dabei verwendeten theoretischen Grundlagen und getroffenen Annahmen. Zur Implementierung und der Ableitung der dargestellten Theorie in die FEM Umgebung wird auf FEM Literatur verwiesen [6, 17, 19].

\subsection{Materialmodelle}

\subsubsection{Struktur}

Für das strukturelle System wird die Struktur durch die Differentialgleichung der Bewegung für einen Kontinuumskörper beschrieben, wobei kleine Deformationen angenommen werden. Ausgehend von der Navier Gleichung im Equilibrium kann nach [19] die Bewegungsgleichung mithilfe des Differenzialoperators B wie folgt in Matrizen Schreibweise dargestellt werden.

$$
\boldsymbol{f}_{V}+B^{T} \sigma=\rho \boldsymbol{a}
$$

mit

$$
\begin{aligned}
B & =\left(\begin{array}{cccccc}
\frac{\partial}{\partial x} & 0 & 0 & 0 & \frac{\partial}{\partial z} & \frac{\partial}{\partial x} \\
0 & \frac{\partial}{\partial y} & 0 & \frac{\partial}{\partial z} & 0 & \frac{\partial}{\partial x} \\
0 & 0 & \frac{\partial}{\partial z} & \frac{\partial}{\partial y} & \frac{\partial}{\partial z} & 0
\end{array}\right)^{T} ; \\
\sigma & =\left(\begin{array}{lll}
\sigma_{11} & \sigma_{12} & \sigma_{13} \\
\sigma_{21} & \sigma_{22} & \sigma_{23} \\
\sigma_{31} & \sigma_{32} & \sigma_{33}
\end{array}\right) ; \quad \boldsymbol{a}=\frac{\partial^{2} u}{\partial t^{2}} ; \boldsymbol{u}=\left(\begin{array}{l}
u_{1} \\
u_{2} \\
u_{3}
\end{array}\right)
\end{aligned}
$$

Wobei $\sigma$ den Spannungstensor nach Cauchy, a die Beschleunigung des Festkörpers und $f_{V}$ die Summe der äußeren Kräfte darstellt. Die Modellierung des strukturellen Materialverhaltens beruht auf der Annahme, dass die Werkstoffe sich linear elastisch und isotrop verhalten. Für solche Materialen lässt sich der Zusammenhang zwischen Spannungen und Verzerrungen über das Hooksches Gesetz definieren. Die berücksichtigte innere Dämpfung des Materials wird über ein komplexes E-Modul beschrieben. Dieses Modell entspricht einer Strukturdämpfung mittels isotropen Verlustfaktors $\eta$. Dabei kann das komplexe E-Modul E des Materials wie folgt beschrieben werden:

$$
E=E(1+i \eta)
$$

\subsubsection{Luft}

Die Differenzialgleichung des Fluids kann zur Beschreibung des mechanischen Feldes aus der Bewegungsgleichung, den kinematischen Beziehungen und dem Stoffgesetz unter folgenden Annahmen hergeleitet werden [19]:

- Das Fluid ist nicht viskos

- Das Fluid unterliegt nur kleinen Verschiebungen

- Das Fluid ist rotationsfrei

- Das Fluid ist kompressibel

Damit können die zugrundeliegenden partiellen Differentialgleichungen wie folgt angeschrieben werden:

$$
\frac{\partial^{2} p_{f}}{\partial^{2} t}-c_{0}^{2} \nabla^{2} p_{f}=c_{0}^{2} \frac{\partial q_{f}}{\partial t}
$$

Dabei stellen $p_{f}$ den dynamischen Druck, $q_{f}$ den hinzugefügte Massenfluss und $c_{0}$ die Schallgeschwindigkeit im Fluid dar.

\subsubsection{Dämmstoff}

Eine Modellierung der Schallübertragung über den Hohlraum dieser Konstruktion bedarf somit einer geeigneten Modellierung der Schalltransmission und des akustischen Verhaltens solcher Dämmstoffe. Aufgrund der feingliedrigen Struktur dieser Materialien ist es in der Regel nicht möglich, diese Strukturen und die Wechselwirkung zwischen Luft und Fasern in der FEM aufzulösen. Eine Möglichkeit das Verhalten solcher Materialen in Simulationsmodellen abzubilden ist die Darstellung als äquivalentes Fluid dessen Eigenschaften durch das Fluid in der porösen Struktur und durch die der porösen Struktur selbst bestimmt werden. Die Implementierung des 
äquivalenten Fluids mit dessen Eigenschaften der komplexen Schallgeschwindigkeit $c_{c}$, der äquivalenten Dichte $\rho_{c}$ und des effektiven Kompressionsmoduls $\mathrm{K}_{\text {eff }}$ erfolgt analog zu 3.1.2.

$$
\begin{gathered}
\rho_{\mathrm{c}}=\rho_{\text {rig }} \\
c_{\mathrm{C}}=\sqrt{\frac{K_{\text {eff }}}{\rho_{\text {rig }}}}
\end{gathered}
$$

Die Meisten, der in der Literatur präsentierten porösen Materialmodelle, stellen dabei den Strukturrahmen als starr dar [20]. Einen unterschiedlichen Ansatz bietet [21], in dem die Mikrostruktur des Materials schlaff modelliert wird. In [22] wird ein Vergleich dieser beiden Modellierungsansätze in unterschiedlichen Frequenzbereichen verfolgt. Es konnte allerdings keine klaren Vor- bzw. Nachteile der unterschiedlichen Ansätze herausgearbeitet werden. Das Modell Johnson-Champoux-Allard (JCA), das in der zugrunde liegenden Arbeit verwendet wurde, geht von einer starren porösen Struktur aus [20], die durch die komplexen Kenngrößen der Dichte $\rho_{\text {rig }}$ und des Kompressionsmoduls $\mathrm{K}_{\text {eff }}$ beschrieben wird.

$$
\begin{gathered}
\rho_{\text {rig }}=\frac{\tau_{\infty} \rho_{f}}{\varepsilon_{p}}\left[1+\frac{R_{f} \varepsilon_{p}}{i \omega \rho_{f} \tau_{\infty}} \sqrt{1+\frac{4 i \omega \tau_{\infty}^{2} \mu \rho_{f}}{R_{f}^{2} L_{V}^{2} \varepsilon_{p}^{2}}}\right] \\
K_{\text {eff }}=\frac{\gamma p_{A}}{\varepsilon_{p}}\left[\gamma-(\gamma-1)\left(1+\frac{8 \mu}{i \omega L_{t h}^{2} \operatorname{Pr} \rho_{f}} \sqrt{1+\frac{i \omega L_{\text {th }}^{2} \operatorname{Pr} \rho_{f}}{16 \mu}}\right)^{-1}\right]^{-1} \\
L_{V}=\frac{1}{s} \sqrt{\frac{8 \mu \tau_{\infty}}{\varepsilon_{p} R_{f}}}
\end{gathered}
$$

$T$ ist der Porositätskrümmungsfaktor, $L_{V}$ ist die viskose charakteristische Länge, $L_{\text {th }}$ ist die thermisch-charakterische Länge. Weitere Modelle zur Beschreibung der Schallausbreitung in porösen Medien werden z.B. in [23-26] dargestellt.

\subsection{Schalldämmmaß}

Das Schalldämmmaß (oder Schalltransmissionsverlust) einer Wand ist wie folgt definiert:

$$
R=\log _{10}\left(\frac{1}{\tau}\right)
$$

Der Schalltransmissionsgrad $\tau$ ist das Verhältnis aus der durch das Schallfeld in einem Senderaum resultierenden auf die Wand einfallenden Schallintensität $I_{i}$ und der von der Wand in einen Empfangsraum abgestrahlten Schallintensität $l_{\mathrm{e}}$.

$$
\tau=\frac{\operatorname{Re}\left(l_{i}\right)}{\operatorname{Re}\left(/ I_{e}\right)}
$$

Diese Definition stellt insbesondere die numerische Simulation vor größere Herausforderungen, da neben der eigentlichen Struktur des zu untersuchenden Bauteils auch Sende- und Empfangsraum und deren akustischen Oberflächeneigenschaften zu berücksichtigen sind. Ebenso ist in der Regel das gemessene Schalldämmmaß nach ÖNORM EN ISO 10140-2:2020 gerade bei tiefen Frequenzen von der Lautsprecherposition und des resultierenden modalen Schallfeldes abhängig. Die Grenzfrequenz für den überlicherweise ca. $50 \mathrm{~m}^{3}$ großen Empfangsraum liegt nach [27] bei ca. $270 \mathrm{~Hz}$. Für eine möglichst fehlerfreie Abbildung der Messsituation ist daher der Senderaum und dessen Eigenschaften ganzheitlich innerhalb der

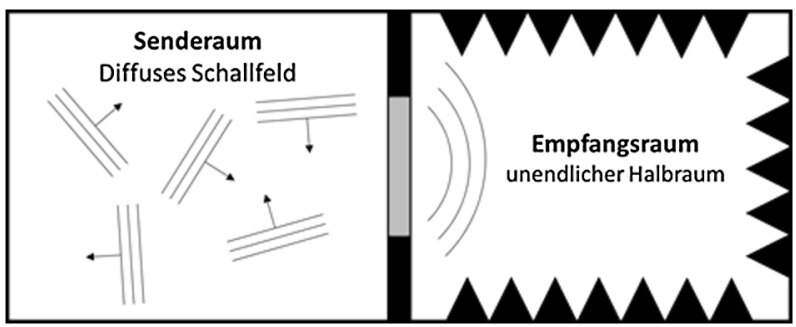

Abb. 2. Schematische Darstellung der innerhalb der FEM Umgebung modellierten Schallfelder in Sende- und Empfangsraum

Simulation abzubilden. Dadurch sind in Relation zu den interessierenden Biegewellenlängen sehr große Domänen zu diskretisieren, wodurch ein hoher Berechnungsaufwand entsteht. Um dieses Problem zu lösen kennt die Literatur verschiedene Ansätze [28, 29]

In dieser Arbeit wird das nicht diffuse Schallfeld im tiefen Frequenzbereich, der Einfluss des Raumes und der Lautsprecherposition nicht berücksichtigt. Das auf das Bauteil einfallende Schallfeld im Senderaum wird als homogenes, isotropes und diffuses Schallfeld beschrieben. Dabei wurde der Ansatz verfolgt, dass das diffuse Schallfeld auf der Senderaumseite des Bauteils als Summe von N unkorrelierten Ebenen Wellen mit gleichförmig zufällig verteilten Einfallswinkeln $\varphi_{n}, \theta_{n}$ beschrieben werden kann. Die Druckverteilung $p_{\text {Senderaum }}(x, y, z)$, als Grundbedingung in der FEM Simulation kann also wie folgt berechnet werden:

$$
\begin{gathered}
p_{\text {Bauteil }}=p_{\text {Raum }}+p_{\text {Reflexion }} \\
p_{\text {Raum }}=\frac{1}{\sqrt{N}} \sum_{n=1}^{N} e^{-i\left(k_{n, x} x+k_{n, y} y+k_{n, z} z\right)} e^{i \Phi_{n}}
\end{gathered}
$$

Unter der Annahme, dass das Bauteil selbst schallhart ist, kann die reflektierende Komponente des Schalldruckfeldes am Bauteil wie folgt berechnet werden:

$$
p_{\text {Raum }}=\frac{1}{\sqrt{N}} \sum_{n=1}^{N} e^{-i\left(k_{n, x} x+k_{n, y} y+k_{n, z} z\right)} e^{i \Phi_{n}}
$$

Mit

$$
\begin{gathered}
k_{n, x}=\cos \left(\theta_{n}\right) \\
k_{n, y}=\sin \left(\theta_{n}\right) \cos \left(\varphi_{n}\right) \\
k_{n, z}=\sin \left(\theta_{n}\right) \sin \left(\varphi_{n}\right)
\end{gathered}
$$

Die Oberflächen des Empfangsraums werden dabei absorbierend modelliert, was bedeutet das die Rückkopplung zwischen Bauteil und an der Empfangsraumoberfläche reflektierten Schallwellen besteht. Damit kann der Empfangsraum als Unendlicher Halbraum modelliert werden. Innerhalb der FEM Umgebung erfolgt dies mittels Luftvolumen, dass mittels PML "Perfect Matched Layer" eingefasst ist. Dieser Rand kann als Absorbierende Randbedingung für die Luftdomäne angesehen werden. Es findet somit keine Reflexion des vom Bauteil abgestrahlten Schallfeldes an dem Rand des Luftvolumens statt. Zur Implementierung in die FEM und der mathematischen Beschreibung der Eigenschaften des PML wird auf [19] verwiesen.

Eine volle Diskretisierung des Raumvolumens auf der Sende- und der Empfangsraumseite ist somit nicht notwendig. Abbildung 2 zeigt eine Schematische Darstellung der Situation, die innerhalb der FEM Umgebung abgebildet wurde. 
Tab. 5. Materialparameter - Schraube

\begin{tabular}{ll}
\hline Bezeichnung & Beschreibung \\
\hline Eingespannt & $\begin{array}{l}\text { Die Plattenränder der Gipsfaserplatte sind eingespannt, es wird keine Verschiebung oder Verdrehung des Randes } \\
\text { zugelassen } \\
\text { Aufgelagert }\end{array}$ \\
$\begin{array}{l}\text { Die Plattenränder der Gipsfaserplatte sind aufgelagert, es wird keine Verschiebung aber eine Verdrehung des Randes } \\
\text { Punktgelagert } \\
\text { Das Bauteil ist nur an den vier Eckpunkten der Gipsfaserplatte gehalten. Die Plattenränder können sich ansonsten frei } \\
\text { bewegen }\end{array}$ \\
\hline
\end{tabular}

Die Anbindung an den Prüfstand und die dadurch beeinflusste Befestigung des Plattenrandes hat einen wesentlichen Einfluss auf das Schwingungsverhalten und somit auf das Schalldämmmaß einer Wandkonstruktion. [4] Bei Modellen unter Berücksichtigung von endlichen Ausmaßen der Bauteildomänen ist es somit erforderlich, die Randbedingungen der Wand zu kennen und dementsprechend zu modellieren. Da die Einbausituation bei derartigen Bauteilen stehts variiert und oft unklar ist, werden in der nachfolgenden Untersuchung verschiedene Anbindungen, entsprechend Tab. 5, an den Prüfstand modelliert.

\subsection{Kopplung Fluid - Struktur}

Grundlegend erfolgt die Kopplung von Struktur und Fluid an der gemeinsamen Grenze ihrer beiden Domänen durch ein vorausgesetztes Kräftegleichgewicht und einer Kontinuitätsbedingung. Die erste Bedingung zur Kopplung von Fluid und Struktur folgt aus dem bedingten Gleichgewicht zwischen Schalldruck $p_{f}$ des Fluids und der Normalkomponente $\sigma_{\mathrm{n}}$ der mechanischen Spannungen der Wand an der Grenzfläche.

$$
\sigma_{n}=-\mathbf{n} p_{f}
$$

Die zweite Bedingung folgt aus der Anforderung der Kontinuität an der Grenze zwischen Struktur und Fluid und bedingt das die Normalkomponenten der Geschwindigkeiten der Strukutroberfläche v und die der des Fluids $v_{f}$ an der Grenzschicht gleich groß sind und kann wie folgt dargestellt werden [19]:

$$
\begin{gathered}
v=\frac{\partial \boldsymbol{u}}{\partial t} v_{f}=-\nabla \psi \\
\boldsymbol{n} \cdot\left(v-v^{\prime}\right)=\mathbf{0} \\
\boldsymbol{n} \cdot \frac{\partial \boldsymbol{u}}{\partial t}=-\boldsymbol{n} \cdot \nabla \psi
\end{gathered}
$$

\subsection{Schraubenmodell}

Die unterschiedlichen Komponenten von Leichtbaukonstruktionen sind in den häufigsten Modellen mittels Schrauben miteinander verbunden. Untersuchungen haben gezeigt, dass diese Schraubverbindungen einen signifikanten Einfluss auf das Schalldämmmaß dieser Konstruktionen besitzen. Die Abbildung dieser Verbindungen in Prognosemodellen ist daher notwendig. Die Literatur bietet unterschiedliche Möglichkeiten der Modellierung der Schraubenverbindung, deren Funktionsprinzipien in Abb. 3 dargestellt sind. Die simpelste Art der Modellierung ist die gesamte gemeinsame Fläche zwischen dem Steher und der Platte als starr verbunden anzusehen (Model a). Am häufigsten wird im tiefen Frequenzbereich von einer linienförmigen Verbindungsmodellierung (Model c) ausgegangen. Bei diesem Modell werden alle Schrauben entlang der Achse eines Stehers zu einer starren Linienverbindung zwischen Steher und Platte zusammengefasst. Erst in höheren Frequenzbereichen des Spektrums $>500 \mathrm{~Hz}$, bei denen der Schraubenabstand im Vergleich zur a)
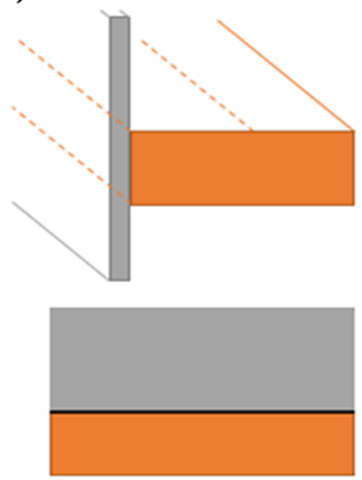

b)

c)

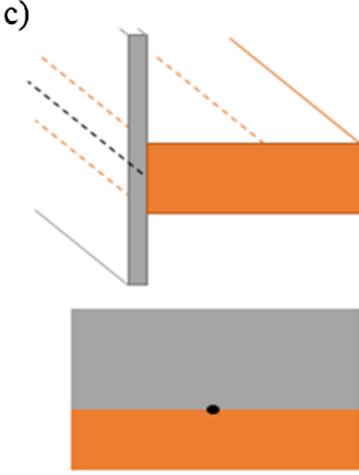

Abb. 3. Darstellung der unterschiedlichen Modellierungsansätze von Schraubverbindungen innerhalb einer FEM Umgebung (a. Fläche, b. Punkt, c. Linie)

Biegewellenlänge auf der Platte größer ist, muss zu einer punktförmigen Modellierung (Modell b) übergegangen werden. Dabei wird die Schraubverbindung an deren tatsächliche Lage mittels starrer punktueller Verbindung modelliert. In [24] wurde gezeigt, dass durch eine Modellierung der Schraubenverbindung, als fixe punktuelle Verbindung (Modell b), die Modellierung zu einem zu steifen Verhalten führt. Weiteres wurde in [4] gezeigt, dass unterschiedliche Schraubengeometrie wie z.B. Schraubenkopfdurchmesser $d_{w}$, Schraubenlänge $I_{f}$ und Gewindesteigung $P$ erheblichen Einfluss auf die zu erwartende Steifigkeit und Schwingungsverhalten der Konstruktion führen. In [4, 5] konnte gezeigt werden, dass folgende Parameter einen wesentlichen Einfluss auf das Schalldämmmaß einer Leichtbaukonstruktionen haben können:

- Schraubenposition

- Schraubenabstand

- Materialeigenschaften

- Anzugsmoment 


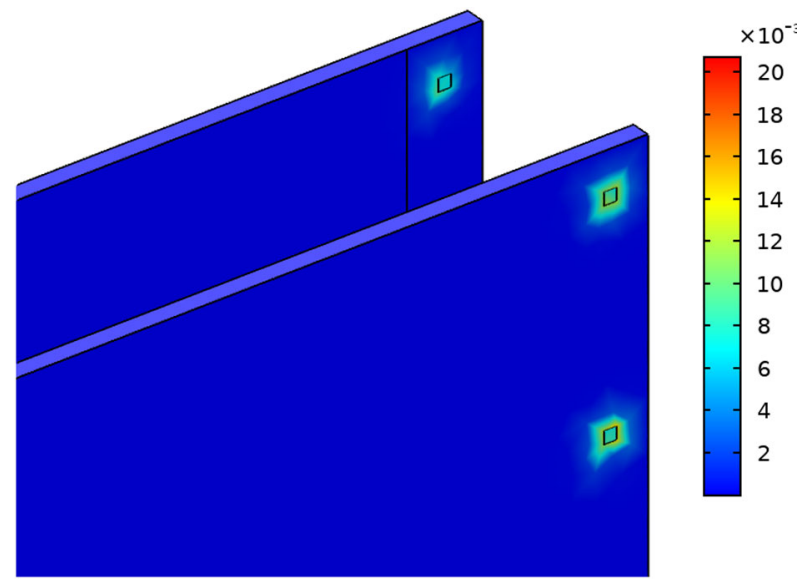

Abb. 4. Ergebnis der stationären Untersuchung in Form der Spannungsverteilung in der Plattenstruktur unter Belastung durch die Vorspannung der Schraubenelemente in $\mathrm{N} / \mathrm{mm}^{2}$

Keines der in Abb. 3 dargestellten Modelle ermöglicht die Abbildung des Einflusses des Schraubenanzugsmoments auf das Schalldämmmaß des Bauteils. Hierzu wurde ein Modell entwickelt die alle der genannten Einflussparameter berücksichtigt. In [4] wurde ein Modell entwickelt, dass die Schraube als Kombination aus 2D und 1D Elementen abbildet. Das Anzugsmoment der Schraube und die dadurch resultierende Anpressung der Platte an den Steher wird dabei als punktuelle Kraft auf das Bauteil aufgebracht. Dieser Ansatz besitzt Nachteile bei der Simulation. Die Einzelkraft, regt in der Simulation das Bauteil bei der Simulation in der Frequenzdomäne sinusförmig an und weicht dadurch von der physikalischen Wirkung des Schraubenanzugsmoments ab. Das in dieser Arbeit modifizierte Modell, bei dem die Schraube durch einen vorgespannten Quader, dessen zwei parallel zu Plattenebene verlaufenden Oberflächen steif mit Platte und Steher verbunden sind, umgeht dieses Problem. Die Berücksichtigung des Schraubenanzugsmoments erfolgt durch eine in der Symmetrie Achse wirkende Normalspannung in Schraubenrichtung. Diese Normalspannung kann aus dem Anzugsmoment M und der Gewindesteigung P resultierenden Normalkraft Fvorspannung berechnet werden.

$$
F_{\text {Vorspannung }}=\frac{M}{0.16 \cdot P}
$$

Der stationäre Spannungszustand in der Struktur, dargstellt in Abb. 4, hervorgerufen durch diese Vorspannung, dient als Ausgangszustand für die Berechnung des Schalldämmmaßes in der Frequenzdomäne. Zwischen Platte und Steher findet abgesehen von dieser Verbindung keine Kraftübertragung statt. Die Materialeigenschaften dieses Quaders werden aus dem Produkt der Materialeigenschaften der Schraube und dem Querschnittsverhältnis zwischen Schraube und Quader gebildet. Die Größe des Quaders richtet sich nach der verwendeten minimalen Elementgröße in der Diskretisierung der Struktur. In den dargestellten Arbeiten hat der Quader somit eine Querschnittsfläche von $1 \mathrm{~cm} \times 1 \mathrm{~cm}$.

\subsection{Geometrie}

Die in Abschn. 2 beschriebene Struktur wurde innerhalb der FEM Umgebung COMSOL entsprechend Abb. 5 modelliert und wie in Abb. 6 dargestellt diskretisiert. Abgesehen von der Schraubenmodellierung sind keine Vereinfachungen hinsichtlich der tatsächlichen Geometrie getroffen worden

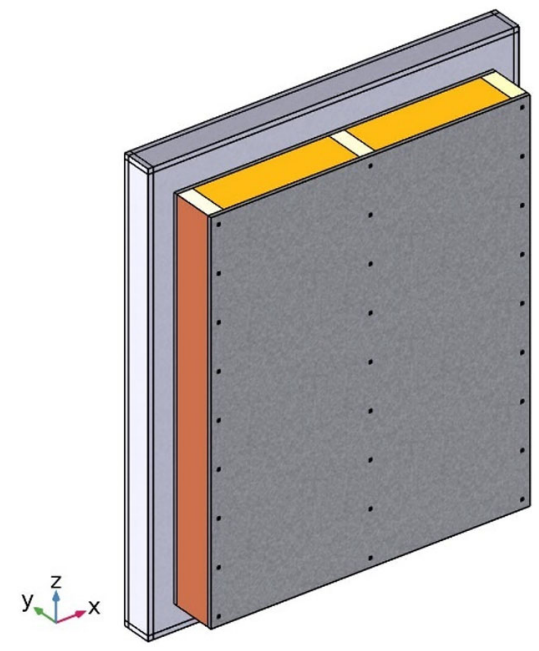

Abb. 5. Darstellung der innerhalb der FEM Umgebung abgebildeten Geometrie des untersuchten Bauteils und des Luftvolumens auf der Empfangsraumseite

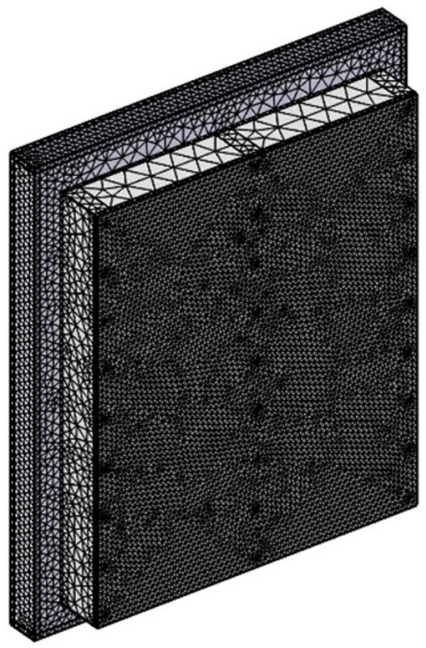

Abb. 6. Darstellung der innerhalb der FEM Umgebung diskretisierten Geometrie des untersuchten Bauteils und des Luftvolumens auf der Empfangsraumseite

Abbildung 6 zeigt das resultierende Netz innerhalb der FEM Umgebung in der Einstellung "normal", dass zur Berechnung der Unbekannten in allen Berechnungsschritten (Spannungsverteilung in der Struktur aufgrund der Schraubenvorspannung, Verschiebung der Struktur und Druckverteilung im Fluid aufgrund des Einfallenden Schallfelds). In Table 6 sind die unterschiedlichen Einstellungen der verwendeten Berechnungsgitter dargestellt, die für die anschließende Netzsensitivitätsanalyse herangezogen werden.

\section{Numerische Ergebnisse}

\subsection{Validierung}

Zur Validierung wurden Messdaten herangezogen die in dem Projekt "Schall.Holz.Bau” [5] gewonnen wurden. Im Rahmen des Projektes wurde untern anderem der Einfluss des Schraubenabstandes auf das Schalldämmaß von Holzleichtkonstruktionen untersucht. Das frequenzabhängige Schalldämmaß entsprechend EN ISO 
Tab. 6. Parameter zur Beschreibung des Netzes zur Diskretisierung des Bauteils in der FEM Umgebung

\begin{tabular}{lllc}
\hline Bezeichnung & $\begin{array}{l}\text { Min. } \\
\text { Elementgröße } \\
\text { in } \mathrm{m}\end{array}$ & $\begin{array}{l}\text { Max. } \\
\text { Elementgröße } \\
\text { in } \mathrm{m}\end{array}$ & $\begin{array}{l}\text { Gesamtanzahl } \\
\text { der Elemente }\end{array}$ \\
\hline Coarser & 0.068 & 0.323 & 24759 \\
Coarse & 0.0476 & 0.255 & 43471 \\
Normal & 0.0306 & 0.17 & 77513 \\
Fine & 0.017 & 0.136 & 117264 \\
\hline
\end{tabular}

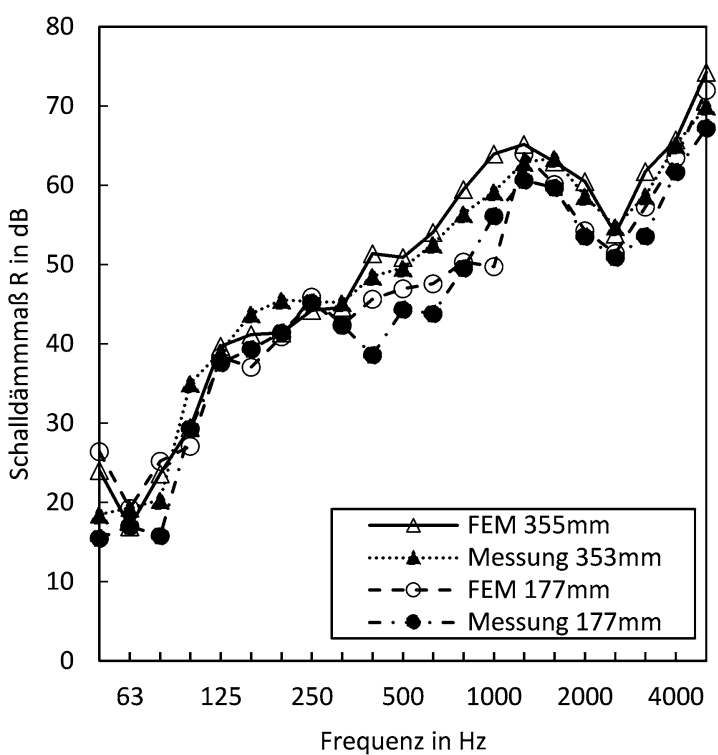

Abb. 7. Vergleich des berechneten und gemessenen frequenzabhängigen Schalldämmmaßes der untersuchten Struktur mit $177 \mathrm{~mm}$ und $355 \mathrm{~mm}$ Schraubenabstand

10140-2:2020 der in Abschn. 2 beschriebene Struktur wurde dabei in einem Fensterprüfstand gemessen. Abbildung 7 und 8 zeigt den Vergleich von Messung und Berechnung des frequenzabhängigen Schalldämmmaßes für die Schraubenabstände $177 \mathrm{~mm}$ und $355 \mathrm{~mm}$. Im tiefen Frequenzbereich $<100 \mathrm{~Hz}$ sind insbesondere mit dem Schraubenabstand von $177 \mathrm{~mm}$ Abweichungen von bis zu $10 \mathrm{~dB}$ zu erkennen. Da das Schalldämmmaß in diesem Frequenzbereich bei der dieser Konstruktion wesentlich von den Einbaubedingungen abhängt wird vermutet, dass die Abweichung, mit der in der Simulation nicht exakt nachgebildeten Montagesituation begründet werden kann. Für weitere Untersuchungen in diesem Frequenzbereich wird vorgeschlagen, dass Montagebedingungen gewählt werden, die zwar von den angewendeten Normvorgaben abweichen, aber in numerischen Simulationen exakter abbildbar sind. Im Frequenzbereich $>100 \mathrm{~Hz}$ bis $400 \mathrm{~Hz}$ stimmen für beide Schraubenabstände die beiden Kurvenverläufe sehr gut überein und die Abweichungen sind kleiner als $2 \mathrm{~dB}$. Der Vergleich in Abb. 7 zeigt, den Einfluss des Schraubenabstandes im Frequenzbereich um $500 \mathrm{~Hz}$. In [4] wurde gezeigt, dass diese Unterschiede durch Beeinflussung des Abstrahlgrades und der Unterdrückung von Eigenmodenformen der Platte erklärbar sind. Diese Phänomene begründen den Einbruch bei $500 \mathrm{~Hz}$ und können offensichtlich nicht exakt, mit Abweichungen von bis zu $6 \mathrm{~dB}$, durch das Modell abgebildet werden. Über $500 \mathrm{~Hz}$ ist das Modell sehr gut in der Lage den Kurvenverlauf des Schalldämmmaßes nachzuvollziehen. Auch der deutlich erkennbare

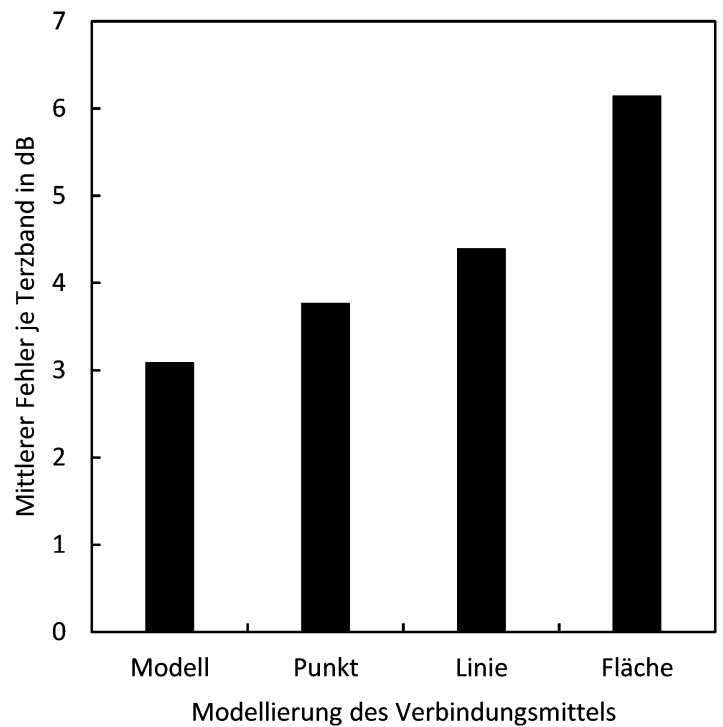

Abb. 8. Über die Terzbänder von $50-5000 \mathrm{~Hz}$ arithmetische gemittelte Differenz zwischen Messung und Berechnung des frequenzabhängigen Schalldämmmaßes der untersuchten Struktur mit $177 \mathrm{~mm}$ Schraubenabstand - Vergleich unterschiedlicher Modellierungsansätze zur Abbildung der Verbindungsmittel zwischen Platte und Steher

Koinzidenzeinbruch ist in der Simulation mit Abweichungen zu den Messergebnissen von $<4 \mathrm{~dB}$ sehr gut zu erkennen.

\subsection{Modellvergleich - Verbindungsmittel}

Abbildung 8 zeigt den Vergleich des einzahlbewertenden Schalldämmmaßes der Messung (Schraubenabstand $177 \mathrm{~mm}$ ) und der Berechnung entsprechend Abschn. 3.2 mit unterschiedlichen Modellen für die Schraubverbindung zwischen Platte und Steher. Einer der größten Einflussparameter der Verbindung zwischen Steher und Platte ist der Schraubenabstand [4]. Damit ist auch in der dargestellten Untersuchung erklärbar, dass die Modelle, die den Schraubenabstand und die Schraubenposition berücksichtigen ("Modell" und "Punkt") die beste Übereinstimmung zwischen Messung und Berechnung besitzen. Weiters ist zu erkennen, dass das in Abschn. 3 vorgestellte Modell, inklusive Berücksichtigung des Schraubenanzugsmoments, die geringste Abweichung von ca. $3 \mathrm{~dB}$ im Einzahlbewerteten Schalldämmmaß aufweist.

Abbildung 9 zeigt den Vergleich der ermittelten Differenz zwischen dem gemessenen und berechneten frequenzabhängigen Schalldämmmaß. Die Berechnungen wurden mit den in Abschn. 3.2 beschriebenen unterschiedlichen Schraubenmodellen durchgeführt. Der Vergleich zeigt, dass die große Abweichung zwischen Modellund Messergebnis im Frequenzbereich $<100 \mathrm{~Hz}$ nur geringfügig durch die Art des Modells der Schraubverbindung beeinflusst wird. Womit bekräftigt werden kann, dass in diesem Bereich die Montagebedingungen des Prüfkörpers im Prüfstand hier der wesentlicher Einflussgeber ist, der in der Simulation aufgrund der Komplexität nicht korrekt wiedergegeben werden konnte. Oberhalb von $100 \mathrm{~Hz}$ zeigt das Modell, außer in dem bereits diskutierten Frequenzbereich um $500 \mathrm{~Hz}$, eine sehr gute Übereinstimmung von $0 \mathrm{~dB}-4 \mathrm{~dB}$ zu den Messdaten. Über den gesamten Frequenzbereich ist zudem zu beobachten, dass das vorgestellte Modell zu Abbildung der Schraubverbindungen die geringste Abweichung zu den Messergebnissen mit durchschnittlich $3 \mathrm{~dB}$ aufweist. 


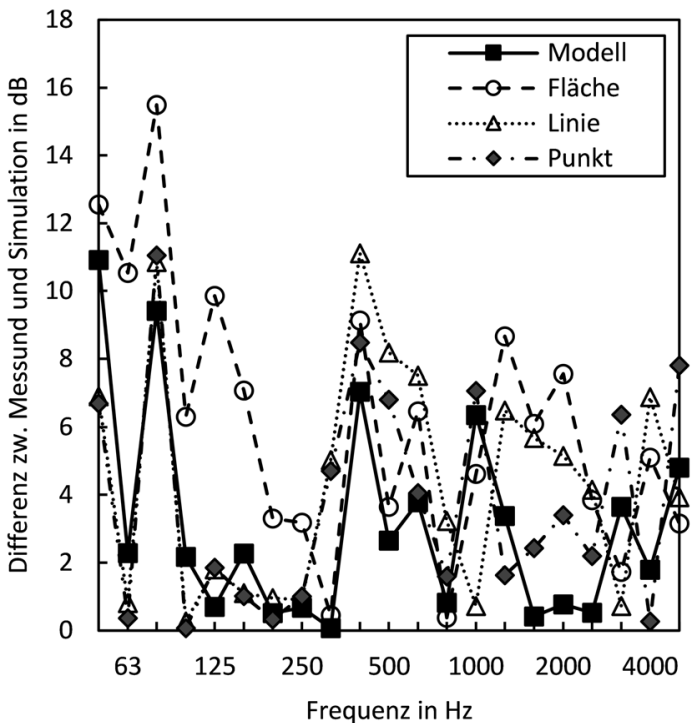

Abb. 9. Differenz zwischen Messung und Berechnung des frequenzabhängigen Schalldämmmaßes der untersuchten Struktur mit $177 \mathrm{~mm}$ Schraubenabstand - Vergleich unterschiedlicher Modellierungsansätze zur Abbildung der Verbindungsmittel zwischen Platte und Steher

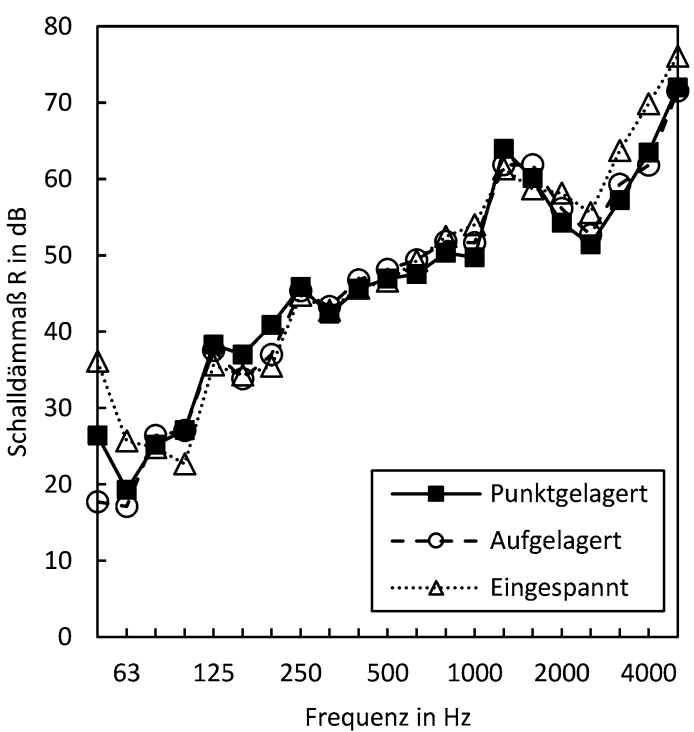

Abb. 10. Frequenzabhängiger Verlauf des berechneten Schalldämmmaßes - Darstellung des Einflusses unterschiedlicher Randbedingungen zur Abbildung der Montage des Prüfkörpers in den Prüfstandsrahmen auf das Berechnungsergebnis

\subsection{Parameterstudie}

Um die Auswirkungen dreier wichtiger Parameter, die das Schalldämmmaß einer Leichtbaukonstruktion beeinflussen, auf die Simulationsergebnisse zu untersuchen wurde eine Parameterstudie für die Montagebedingung, das dynamische E-Modul des Plattenwerkstoffes und des Strömungswiderstandes des Dämmstoffes durchgeführt. Abbildung 10 zeigt die Berechnungsergebnisse des Simulationsmodells mit unterschiedlichen Randbedingungen an den Plattenrändern zur Abbildung der Montagesituation. Gut erkennbar ist, dass mit steigender Steifigkeit der Randbedingung das Schalldämm-

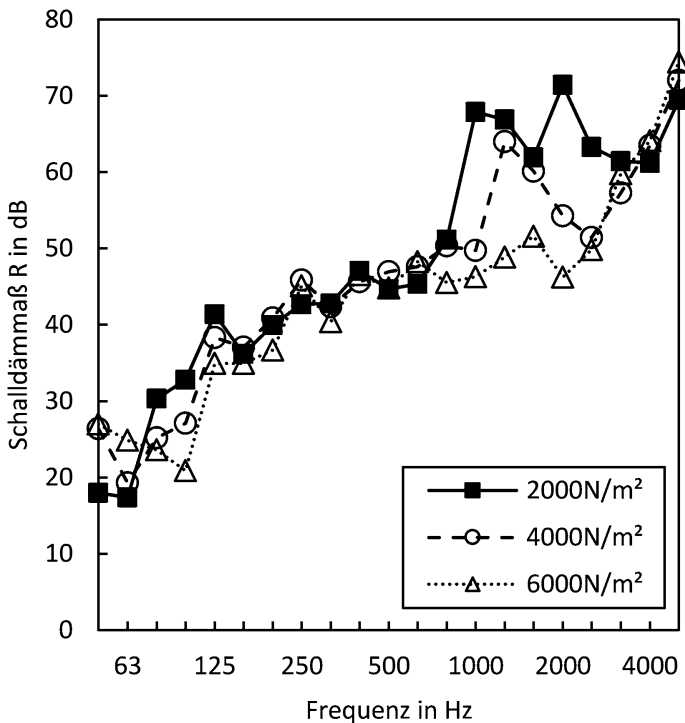

Abb. 11. Frequenzabhängiger Verlauf des berechneten Schalldämmmaßes - Darstellung des Einflusses des dynamischen E-Moduls des Plattenmaterials auf das Berechnungsergebnis

maß im Frequenzbereich $<100 \mathrm{~Hz}$ steigt. Dieses Verhalten ist in der Ligatur durch Messungen [4] bestätigt worden und wird durch das Modell plausibel abgebildet. Oberhalb von $100 \mathrm{~Hz}$ findet kaum eine Beeinflussung des Schalldämmmaßes durch die Randbedingung statt

Abbildung 11 vergleicht die unterschiedlichen Berechnungsergebnisse mit variierenden dynamischen E-Modul des Plattenwerkstoffs $\left(2000 \mathrm{~N} / \mathrm{mm}^{2}-6000 \mathrm{~N} / \mathrm{mm}^{2}\right.$ ). Erkennbar ist, dass sich der Koinzidenzgrenzfrequenz $f_{c}$ mit steigendem E-Modul in Richtung höhere Frequenzen verschiebt. Diese Verschiebung ist plausibel und kann mittels folgenden Ausdrucks auch nachvollzogen werden:

$$
f_{c}=\frac{c_{l}^{2}}{2 \pi} \sqrt{\frac{m^{\prime \prime}}{B}}
$$

Durch die Veränderung der Biegesteifigkeit der Platte wird neben der Lage der Konzidenzgrenzfrequenz im Spektrum auch die Steifigkeit des gesamten Wandsystems beeinflusst. Das ist vorallem im Bereich $<100 \mathrm{~Hz}$ erkennbar. Hier hat der Wandaufbau mit der größten Steifigkeit das höchste Schalldämmmaß.

In Abb. 12 ist der Einfluss des Dämmstoffes im Berechnungsmodell dargstellt. Es werden Materialeigenschaften von drei im Bauwesen typischen Dämmstoffen (Mineralwolle, Steinwolle, EPS) verglichen. Erkennbar ist, dass sich der Masse-Feder-Masse Resonanzeinbruch im Bereich von 50-200 Hz mit der aus den Materialeigenschaften resultierenden Steifigkeit im Frequenzspektrum verschiebt. Hierbei liegt der Einbruch bei der Variante mit dem steifesten Dämmstoff (EPS) am höchsten im betrachteten Spektrum. Oberhalb dieses Einbruchs findet nur eine geringfügige Beeinflussung durch den Dämmstoff statt. Nur die EPS Variante (34000 Pa.s/m²; $115 \mathrm{~kg} / \mathrm{m}^{3}$ ) zeigt im Bereich des Koinzidenzeinbruchs eine Erhöhung des Schalldämmmaßes. Dieses Verhalten ist mit derzeitigem Wissensstand nicht erklärbar. Literaturergebnisse zeigen bei Doppelschaligen Wänden mit steifen Dämmmstoffen zu Hohlraumbedämpfung eher eine verschlechterung des Schalldämmmaßes oberhalb der Masse-Feder-Masse Resonanz. [30] 


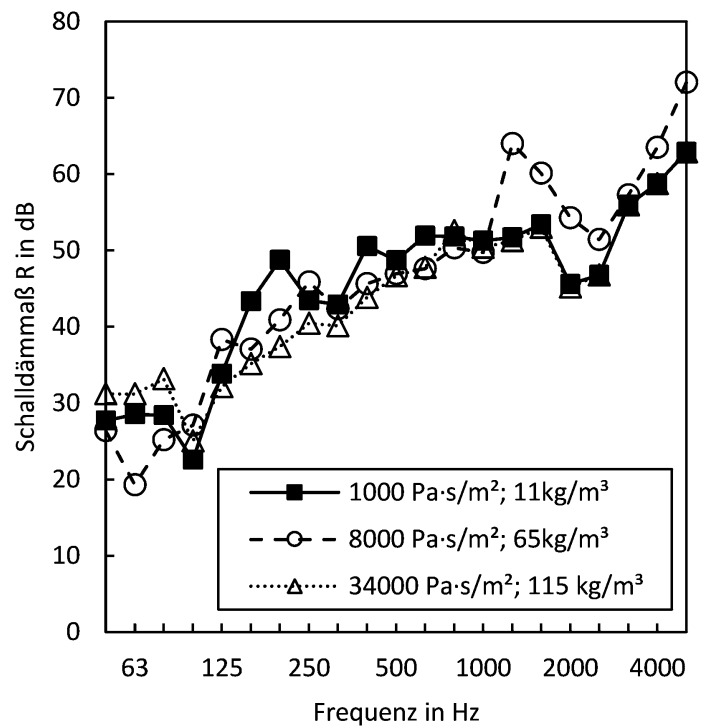

Abb. 12. Frequenzabhängiger Verlauf des berechneten Schalldämmmaßes - Darstellung des Einflusses der Dämmstoffeigenschaften auf das Berechnungsergebnis

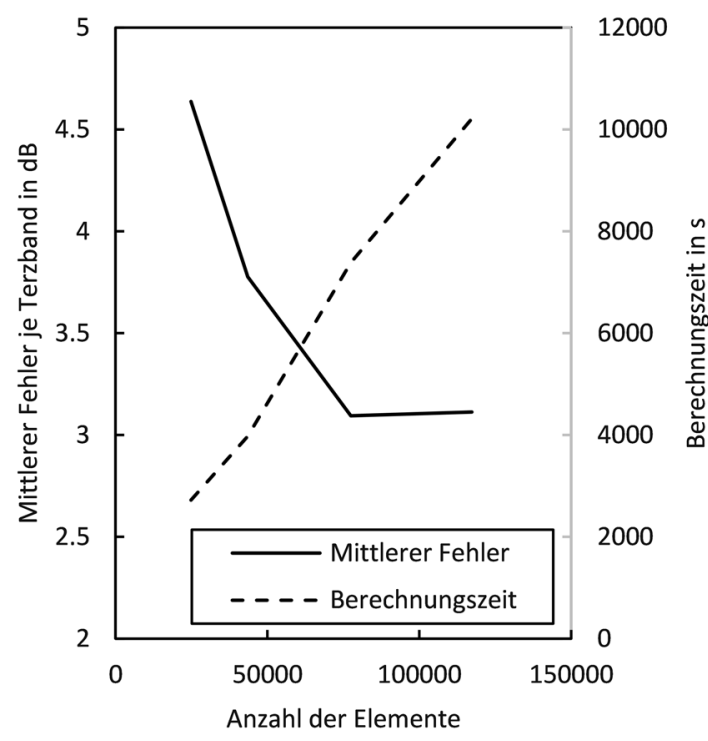

Abb. 13. Zusammenhang zwischen Anzahl der Elemente zur Diskretisierung des Bauteils innerhalb der FEM Umgebung, Berechnungszeit und des mittleren Fehlers je Terzband

4.4 Berechnungseffizienz

Aufgrund der Modellkomplexität bei Finiten Elemente Modellen stellt sich stets die Frage nach dem Zusammenhang zwischen Berechnungsaufwand und zu erwartenden Fehler. Der Berechnungsaufwand hängt stark mit der Anzahl der zur Diskretisierung der Berechnungsdomänen verwendeten Elemente zusammen. Dieser Zusammenhang ist in Abb. 13 dargestellt. Ab ca. 80000 Elementen und einer damit minimalen Elementgröße von $0.03 \mathrm{~cm}$ in der Plattenebene (in Plattendickenrichtung wurden stets 3 Elemente verwendet) ist keine weitere Reduktion der Differenz zwischen Mess- und Berechnungsergebnis zu beobachten. Die Berechnungszeit steigt in dem Betrachteten Bereichs der Feinheit der Diskretisierung linear mit der Anzahl der Elemente an.

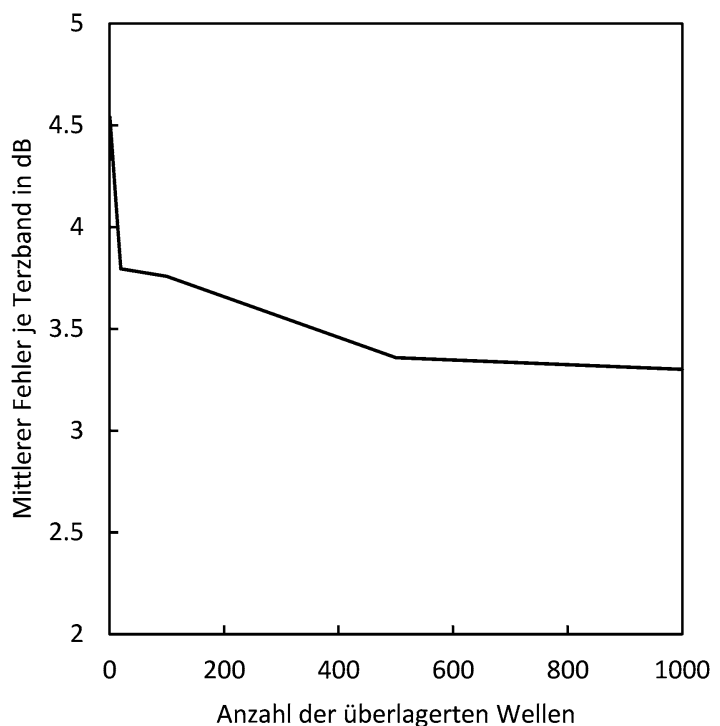

Abb. 14. Zusammenhang zwischen Anzahl der ebenen überlagerten Wellen (Formel X) und der mittleren Differenz zwischen Messung und Berechnung je Terzband

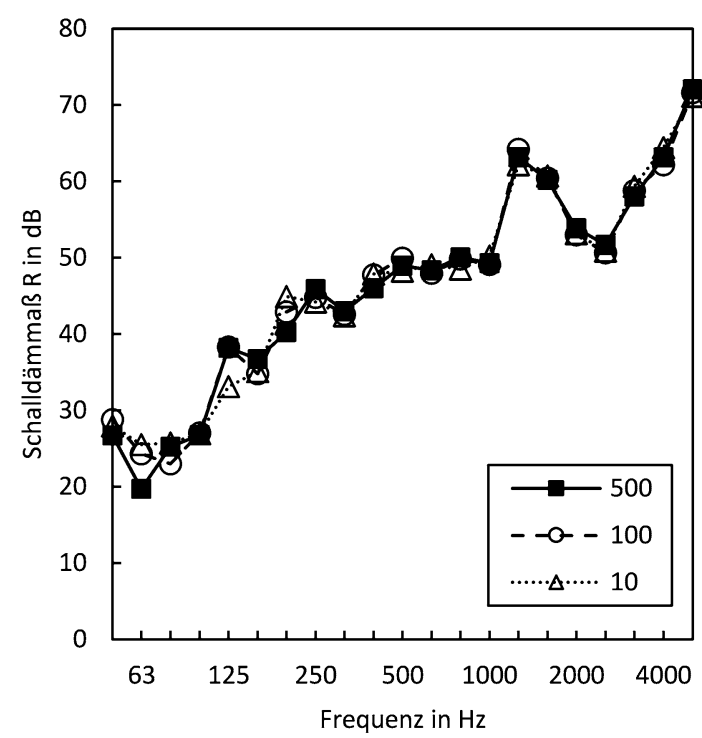

Abb. 15. Frequenzabhängiger Verlauf des berechneten Schalldämmmaßes - Darstellung des Einflusses der Anzahl der ebenen überlagerten Wellen auf das Berechnungsergebnis

Zu der mit höherer Elementanzahl zunehmenden Berechnungszeit kommt in dem beschriebenen Modell hinzu, dass das auf das Bauteil einfallende Schallfeld durch eine Überlagerung einer endlichen Summe von Ebenen unkorrelierten Wellen beschrieben wird. Der Berechnungsaufwand dieser Summe stellt somit eine weitere Berechnungszeit beeinflussende Größe dar. Der Zusammenhang zwischen Anzahl der Wellen, also Summanden in der Summe in Abschn. 3.2, und des mittleren Fehlers je Terzband (Differenz zwischen Messung und Rechnung) ist in Abb. 14 dargestellt. Es ist zu erkennen, dass ab 500 überlagerten Wellen kaum noch eine Verringerung des mittleren Fehlers zu erzielen ist. In Abb. 15 ist zu erkennen, dass die Steigerung der Anzahl der Wellen fast ausschließlich im Frequenzbereich $<200 \mathrm{~Hz}$ auswirkt. 


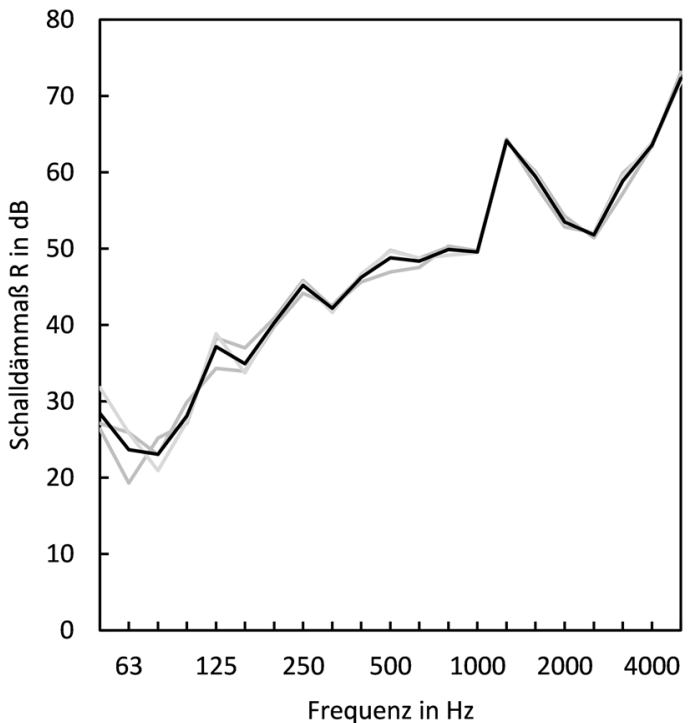

Abb. 16. Frequenzabhängiger Verlauf des berechneten Schalldämmmaßes - Darstellung des Einflusses unterschiedlicher Seeds (Startwert für die Pseudo Zufallszahlenermittlung) auf das Berechnungsergebnis

Ein weiterer Parameter, der das Berechnungsergebnis beeinflusst ist, der Seed mit dem der Zufallszahlengenerator die Winkel und Phasen der einfallenden ebenen Wellen bestimmt. Die dadurch erzeugten pseudo Zufallszahlen besitzen mit jedem sich änderten Seed einen anderen Startwert. Der Einfluss der Variation des Seeds und der damit generierten unterschiedlichen Sets an überlagerten Ebenen Wellen auf das Berechnungsergebnis ist in Abb. 16 dargestellt. Durch das große Verhältnis von Schallwellenlänge zu Bauteilgeometrie ist wie erwartet die Variabilität durch verändertes auf das Bauteil einfallendes Schallfeld bei tiefen Frequenzen $<200 \mathrm{~Hz}$ größer als bei hohen Frequenzen.

\section{Fazit}

Der präsentierte Ansatz zur Modellierung von Schraubverbindungen in FEM Simulationsmodellen zur Prognose des Schalldämmmaßes von Leichtbaukonstruktionen mit unterschiedlichen Verbindungsmittelkonfigurationen konnte das einzahlbewertete Schalldämmmaß einer Gipskartonständerkonstruktion mit einer max. Abweichung zu den Messergebnissen von $3 \mathrm{~dB}$ berechnen. Im Vergleich zu bereits in der Literatur bekannten Modellen bietet das gezeigte Modell die Möglichkeit der Berücksichtigung von unterschiedlichen Schraubenanzugsmomenten. Dieser Parameter ist für Leichtbaukonstruktionen wesentlich und kann unterschiede von bis zu $5 \mathrm{~dB}$ im einzahlbewerteten Schalldämmmaß bedingen [5].

Die detaillierte Beschreibung der Wand innerhalb der FEM Simulationsumgebung ermöglicht die Prognose des Schalldämmmaßes bei Änderung ihrer Eigenschaften und Geometrie. So können Schraubensteifigkeit, Schraubenabstand, Schraubenanzugsmoment, Schraubenposition, Platten-/Stehergeometrie und Dämmstoff im Hohlraum variiert und die Auswirkungen auf das Schalldämmmaß prognostiziert werden. Damit können auch Unsicherheiten auf der Ebene der Materialeigenschaften in diese Prognose einfließen. Für Hersteller und Planer wird neben einem numerischen Optimierungsprozesses und einer Prognose der relevanten bauakustischen Kenngrößen von Trennwänden somit eine Möglichkeit geboten, Maßnahmen zur Steigerung der Verarbeitungsqualität in den Nach- weis der schallschutztechnischen Anforderungen von Baukonstruktionen einfließen zu lassen.

Funding Note Open access funding provided by TU Wien (TUW).

Hinweis des Verlags Der Verlag bleibt in Hinblick auf geografische Zuordnungen und Gebietsbezeichnungen in veröffentlichten Karten und Institutsadressen neutral.

Open Access Dieser Artikel wird unter der Creative Commons Namensnennung 4.0 International Lizenz veröffentlicht, welche die Nutzung, Vervielfältigung, Bearbeitung, Verbreitung und Wiedergabe in jeglichem Medium und Format erlaubt, sofern Sie den/die ursprünglichen Autor(en) und die Quelle ordnungsgemäß nennen, einen Link zur Creative Commons Lizenz beifügen und angeben, ob Änderungen vorgenommen wurden. Die in diesem Artikel enthaltenen Bilder und sonstiges Drittmaterial unterliegen ebenfalls der genannten Creative Commons Lizenz, sofern sich aus der Abbildungslegende nichts anderes ergibt. Sofern das betreffende Material nicht unter der genannten Creative Commons Lizenz steht und die betreffende Handlung nicht nach gesetzlichen Vorschriften erlaubt ist, ist für die oben aufgeführten Weiterverwendungen des Materials die Einwilligung des jeweiligen Rechteinhabers einzuholen. Weitere Details zur Lizenz entnehmen Sie bitte der Lizenzinformation auf http://creativecommons.org/licenses/by/4.0/deed.de.

\section{Literatur}

1. Héroux, M. E., Verbeek, J. (2018): Results from the search for available systematic reviews and meta-analyses on environmental noise. Copenhagen: WHO Regional Office for Europe.

2. OIB-Richtlinie 5 "Schallschutz", April 2019

3. Ribeirinho, M., et al. (2020): The next normal in construction: how disruption is reshaping the world's largest ecosystem. McKinsey \& Company.

4. Neusser, M. (2017): Analyse des Einflusses von Verbindungsmitteln auf das schalldämmmaß von leichten Trennwandkonstruktionen durch realitätsnahe Modellierung von Schraub- und Klebeverbindungen. Dissertation, Technische Universität Wien.

5. Neusser, M., Müllner, H., Exel, R., Smertnig, M. (2019): Schall.Holz.Bau - Bauakustische Optimierung von Holzbau-Außenwänden, St. Pölten.

6. Hongisto, V. (2006): Sound insulation of double panels - comparison of existing prediction models. Acta Acust. Acust., 92, 61-78.

7. Ottosen, N., Petersson, H. (1992): Introduction to the finite element method. New York: Prentice Hall.

8. Pietrzyk, A. (1997): Sound insulation at low frequencies. Chalmers University of Technology, Sweden.

9. Maluski, S. P. S., Gibbs, B. M. (2000): Application of a nite-element model to lowfrequency sound insulation in dwellings. J. Acoust. Soc. Am., 108, 1741-1751.

10. Davidsson, P., Sandberg, G. (2003): Sound reduction in double walls at low frequencies a parametric study. In Proceedings of tenth international congress on sound and vibration, Stockholm, Sweden (8 pages).

11. Kropp, W., Pietrzyk, A., Kihlman, T. (1994): On the meaning of the sound reduction index at low frequencies. Acta Acust., 2, 379-392.

12. Gladwell, G. M. L. (1966): A variational formulation of damped acousto-structural vibration problems. J. Sound Vib., 4, 172-186.

13. Craggs, A. (1973): An acoustic finite element approach for studying boundary flexibility and sound transmission between irregular enclosures. J. Sound Vib., 30, 343-357.

14. Sandberg, G. (1986): Finite Element modelling of fluid-structure interaction. PhD thesis, TU, Lund.

15. Pietrzyk, A., Kropp, W., Kihlman, T.: The Finite Element Method as a tool for the description of sound fields at low frequencies. In InterNoise' 93.

16. Poblet-Puig, et al. (2009): The role of studs in sound transmission. Acta Acust. Acust., 95, 555-567.

17. Arjunan, A., Wang, C. J., Yahiaoui, K., Mynors, D. J., Morgan, T., English, M. (2013): Finite element acoustic analysis of a steel stud based double-leaf wall. Build. Environ., 67, 202-210.

18. Davidson, P. (August 2004): Structure-acoustic analysis; finite element modelling and reduction methods. PhD Thesis, Lund, Sweden.

19. Kaltenbacher, M. (2015): Numerical simulation of mechatronic sensors and actuators. Berlin: Springer.

20. Allard, J. F., Atalla, N. (2009): Propagation of sound in porous media - modelling sound absorbing materials. 2. Aufl. Chichester: Wiley.

21. Göransson, P. (1995): Acoustic finite element formulation of a flexible porous material a correction for inertial effects. J. Sound Vib., 185(4), 559-580.

22. Panneton, R. (2007): Comments on the limp frame equivalent fluid model for porous media. J. Acoust. Soc. Am., 122(6), 217-222.

23. Biot, M. A. (1956): Theory of propagation of elastic waves in a fluid-saturated porous solid. I. Low-frequency range. J. Acoust. Soc. Am., 28(2), 168-178. 
24. Wilson, K. (1993): Relaxation-matched modeling of propagation through porous media, including fractal pore structure. J. Acoust. Soc. Am., 94(2), 1136-1145.

25. Dunn, I. P., Davern, W. A. (1986): Calculation of acoustic impedance of multi-layer absorbers. Appl. Acoust., 19(5), 321-334.

26. Mechel, F. P. (1976): Ausweitung der absorberformel von delany and bazley zu tiefen frequenzen. Acustica, 35(3), 210-213.

27. Lerch, R., Sessler, G., Wolf, D. (2009): Technische Akustik. Berlin: Springer.
28. Elliott, S. J., Maury, C., Gardonio, P. (2005): The synthesis of spatially correlated random pressure fields. J. Acoust. Soc. Am., 117(3), 1186-1201.

29. Diaz-Cereceda, C., Hetherington, J., Poblet-Puig Antonio, J. (2011): Rodrıguez-ferran, a deterministic model of impact noise transmission through structural connections based on modal analysis. J. Sound Vib., 330(12), 6. 2801-2817.

30. Stani, M., Müllner, H. (2005): Sound insulation of plasterboard walls and airflow resistivity: an empirical examination with respect to practical applications. In Forum acusticum, Budapest.

\section{Autoren}

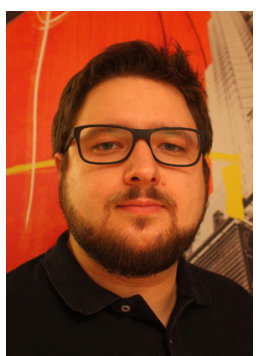

\section{Maximilian Neusser}

schloss 2011 sein Studium des Bauingenieurwesens ab und promovierte 2017 an der Technischen Universität Wien. 2020 wechselte er als Senior Scientist an die Technische Universität Wien. Seine Forschungsschwerpunkte liegen im Bereich der Simulationsund Rechenverfahren in der Bauakustik.

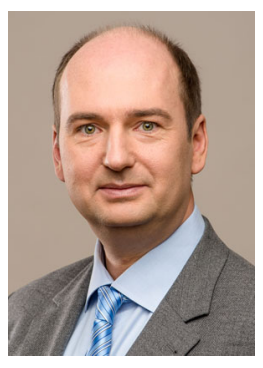

\section{Thomas Bednar}

Arbeitsschwerpunkt ist die Entwicklung von Prognosemethoden für die Planung, Ausführung und den Betrieb von Gebäuden bei der Entscheidung für optimale Maßnahmen zur Vermeidung von Feuchteschäden, den Lastgang für die Infrastruktur aufgrund des Gebäudebetriebs und der Nutzung und die Schallübertragung zur Gestaltung der akustischen Umgebung von Lebewesen besonders Menschen. Österreichisches Mitglied bei mehreren internationalen Forschungskooperationen und Evaluationsgremien, europäischen und internationalen Normungsausschüssen. Leitung des ÖNORMArbeitskreises für die Erstellung von bauphysikalischen Nachweisen, Mitarbeit bei Regelwerken für den Energieausweis und die Bauakustik. Seit 2009 ständiges Mitglied im Bundesdenkmalbeirat des Bundesministeriums für Unterricht, Kunst und Kultur. 